\title{
Anesthetic experience of patients with Fournier's syndrome
}

\author{
Tae Kwan Kim, Jun Rho Yoon, and Yun Kyung Bae \\ Depatment of Anesthesiology and Pain Medicine, Bucheon St. Mary's Hospital, The Catholic University of Korea, Bucheon, Korea
}

Fournier's syndrome is defined as a suppurative bacterial infection of the perineal, genital, or perianal regions. Those conditions often lead to thrombosis of subcutaneous vessels and with infection, resulting in the development of gangrene of the overlying skin and subcutaneous tissue [1]. This rare syndrome is a rapidly progressive and potentially lethal necrotizing fasciitis caused by invasive infections of the lower part of the genitourinary tract, anorectal soft tissue, and genital skin $[1,2]$. The devastating rapidity is typical, as evidenced by the fact that the mean duration of symptoms to become the target of emergency operation is just a few days, and a majority of patients are seriously ill at the time of admission. Anesthetic management of patients with this syndrome is often difficult, due to its devastating nature as well as significant comorbid diseases. However, because of the infrequency of the syndrome, there is limited information regarding the anesthetic management of this disease. We recently encountered the anesthetic management in three cases of patients with Fournier's syndrome. There were three initial emergency and six additional elective operations under general anesthesia, except one spinal anesthesia in an elective case. Therefore, we report these cases and review the relevant literatures.

Immediate and, if required, repetitive operation is important for saving lives in patients with this syndrome [1-3]. Fournier's syndrome is frequently associated with certain diseases and conditions. Diabetes mellitus is probably the most common comorbid disease, as evidenced by our cases [1]. Even when the patient has diabetes, as in our two patients, Fournier's syndrome might be the first clinical disease to be detected. The second common condition is alcoholism, such as in all our patients, because any disorder that compromises the immunity enhances development of a severe infection [1,2]. The other associated clinical features are malnutrition, prolonged hospitalization, radiation therapy, chemotherapy, neurologic deficits, cirrhosis, leukemia, renal failure, organic heart disease, vasculitis, intravenous drug abuse, lupus, cirrhosis, AIDS and steroid medications. In obstetric anesthesia, cervical or pudendal nerve block can induce the syndrome as well [1].

Abnormal laboratory results include hyperglycemia, hypocalcemia, anemia, leukocytosis and thrombocytopenia, as evidenced by our patients [1]. Most of those abnormalities are due to sepsis. The systemic manifestations include fever, tachycardia, and volume depletion similar to those of severe peritonitis [1]. All our patients also had sepsis in terms of the preoperative definition. Two patients looked to be in late distributive shock and the other patient in early distributive shock, respectively. In the case of no active bleeding, delayed or inadequate volume resuscitation is a significant error that would have detrimental effects on the patients's outcome in septic shock. If initial crystalloid fluid resuscitation is insufficient to raise the mean arterial pressure to $65 \mathrm{mmHg}$ and the CVP to 8 to $12 \mathrm{mmHg}$, then vasopressors and inotropes are needed as the second step in the guidelines of early goal-directed therapy [4]. It is rational to use a blood transfusion when the hematocrit is below $30 \%$ when invasive monitoring might be indicated [4]. Among two patients in late septic shock, one patient fortunately responded to our initial fluid resuscitation, whereas the other patient needed dopamine for hypotension. In another patient, early shock occurred, and a blood transfusion and dopamine and norepinephrine were required to achieve an adequate cardiac output and oxygen delivery to maintain vital organ function were needed, because his affected area including

Corresponding author: Jun Rho Yoon, M.D., Depatment of Anesthesiology and Pain Medicine, Bucheon St. Mary's Hospital, The Catholic University of Korea, 2, Sosa-dong, Wonmi-gu, Bucheon 420-717, Korea. Tel: 82-32-340-7075, Fax: 82-32-340-2544, E-mail: pauly@catholic.ac.kr (c) This is an open-access article distributed under the terms of the Creative Commons Attribution Non-Commercial License (http:// creativecommons.org/licenses/by-nc/3.0/), which permits unrestricted non-commercial use, distribution, and reproduction in any medium, provided the original work is properly cited. 
lower extremity was wide and bleeding was ongoing. His septic manifestations reoccurred sporadically over four months of hospitalization and progressed into cardiorespiratory collapse and death after five debridements under general anesthesia.

The preanesthetic investigation of the extent of the lesion is also important, because the ambiguity of the region involved could influence the choice of the anesthetic technique. Koitabashi and colleagues [5] suggested the avoidance of spinal anesthesia in the presence of lumbar subcutaneous gas. Sato and associates [3] recommended that using general anesthesia is preferable for controlling physiologic homeostasis. Fournier's syndrome in particular originateed in anorectal disease, which is the usual subject of regional anesthetic procedures, is known to be aggressive, produces marked systemic toxicity and myonecrosis as in our mortal case, and can be connected with higher mortality [1]. Hence, particular attention should be necessary for the choice of the anesthetic procedures. The reason we performed spinal anesthesia at the secondary wound closure one month after initial debridement in one patient was that he underwent computer tomography to depict the accurate extent of the lesion. Among gravely ill patients it seems wise to not waste precious time doing a lot of investigation to perform regional anesthesia.

The degree of debridement for Fournier's syndrome is variable from simple incision to wide excision with massive bleeding [2]. Many surviving patients require secondary wound closure, skin graft or a reconstructive flap procedure. In spite of appropriate therapy, the mortality rate in Fournier's syndrome is reported to exceed $40 \%$ in many studies [1-3]. Prolonged sepsis manifested by fever or hypotension and lasting for more than 48 hours was experienced among about $40 \%$ of patients, as in our expired patient [2]. Some reports have associated older age, female gender, anorectal causes, delayed admission, the presence of debilitating conditions such as renal failure and hepatic dysfunction with high mortality. Laboratory parameters on admission statistically related to fatality include low hematocrit, calcium, albumin, and cholesterol, and high BUN and alkaline phosphatase levels. The syndrome could rapidly progress into prolonged sepsis, DIC, pneumonia, respiratory failure, diabetic ketoacidosis, renal failure, and heart failure.

In conclusion, our experiences emphasize that Fournier's syndrome has a fatal potential, so optimal conduct of anesthesia requires forethought and sound management as well as an understanding of the pathophysiology of this syndrome for successful anesthesia.

\section{References}

1. Laucks SS 2nd. Fournier's gangrene. Surg Clin North Am 1994; 74: 1339-52.

2. Clayton MD, Fowler JE Jr, Sharifi R, Pearl RK. Causes, presentation, and survival of fifty-seven patients with necrotizing fasciitis of the male genitalia. Surg Gynecol Obstet 1990; 170: 49-55.

3. Sato S, Tomioka T, Orii R, Yamada Y. Anesthetic managements of four patients with Fournier's syndrome. Masui 2008; 57: 355-7.

4. Rivers E, Nguyen B, Havstad S, Ressler J, Muzzin A, Knoblich B, et al. Early goal-directed therapy in the treatment of severe sepsis and septic shock. N Engl J Med 2001; 345: 1368-77.

5. Koitabashi T, Umemura N, Takino Y. A case of Fournier's gangrene contraindicating spinal anesthesia. Anesthesiology 2000; 92: 28990. 